\title{
On pacing trials while scanning brain hemodynamics: The case of the SNARC effect
}

\author{
Sabrina Brigadoi ${ }^{1}$ - Sara Basso Moro ${ }^{2} \cdot$ Roberta Falchi $^{1} \cdot$ Simone Cutini ${ }^{1,3} \cdot$ Roberto Dell'Acqua $^{1,3}$
}

Published online: 16 January 2018

(C) Psychonomic Society, Inc. 2018

\begin{abstract}
Experimental designs used to describe psychological effects on overt human behavior are seldom suited to localize their corresponding neural substrates based on the analysis of stimulus-evoked brain hemodynamic responses. This is because stimuli in behavioral studies are usually separated by intertrial intervals (ITIs) in the order of 1 second or so following a behavioral response, which is notoriously too brief a time to detect a corresponding hemodynamic response. In fact, a solution commonly adopted in neuroimaging studies is to prolong the ITI up to several seconds. In doing so, the consequences of ITI variations between behavioral and neuroimaging design variants are either benignly neglected or explicitly assumed to be negligible. Here, we provide a systematic investigation of the consequence of manipulating ITI in a design optimized to study a well-established and highly replicable psychological phenomenon - the spatial numerical association of response codes (SNARC). The present exploration encompassed standard estimates of the SNARC effect (i.e., on reaction times and accuracy), estimates of ITI effects on the emotional state of participants before and after performing the SNARC task, as well as the degree of perceived task difficulty. The results showed that, in striking contrast to the common wisdom about the nil role of ITI, the substantial number of parametric differences observed between the two ITI conditions suggests that ITI plays a critical role in shaping the meaning of hemodynamic correlate of a psychological, at least the SNARC, effect.
\end{abstract}

Keywords Intertrial interval $\cdot$ Spatial-numerical association of response codes $\cdot$ SNARC $\cdot$ State anxiety

Intertrial interval (ITI) is perhaps the most apparent difference between experimental designs employed to study the effects of a psychological factor on overt human behavior and neuroimaging studies exploring their corresponding neural substrates based on the analysis of stimulus-evoked brain hemodynamic responses measured with functional magnetic resonance imaging (fMRI) or functional nearinfrared spectroscopy (fNIRS). ITIs are usually prolonged in the latter so as to allow the hemodynamic response to a given stimulus to return to baseline prior to collecting the hemodynamic response to a subsequent stimulus. ITI

Sabrina Brigadoi and Sara Basso Moro contributed equally to this work.

Sabrina Brigadoi

sabrina.brigadoi@unipd.it

1 Department of Developmental Psychology, University of Padova, Via Venezia 8, 35131 Padova, Italy

2 Department of Neuroscience, University of Padova, Padova, Italy

3 Padova Neuroscience Center, University of Padova, Padova, Italy lengthening has been employed, for instance, by Brigadoi et al. (2017) to localize the neural circuitry underlying the change-detection task originally designed by Luck and Vogel (1997); by Cutini, Scarpa, Scatturin, Dell'Acqua, and Zorzi (2012) in the magnitude comparison task designed by Dehaene, Bossini, and Giraux (1993); and by Cutini, Scatturin, Basso Moro, and Zorzi (2014) in a task designed by Revkin, Piazza, Izard, Cohen, and Dehaene (2008), to elicit subitizing. ITI lengthening is a solution often implemented uncritically in hemodynamic studies by cognitive neuroscientists, some of them overlooking - or voluntarily dismissing with benign neglect - two critical assumptions on which their findings implicitly rely. One assumption is that ITI plays a negligible role when decoding the neural loci of psychological effects from stimulus-evoked hemodynamic responses. The second assumption is that findings and conclusions about the neural loci of a given psychological effect observed in long-ITI neuroimaging studies generalize to behavioral studies based on short-ITI designs.

The scope of the present study is to test these assumptions through a parametric comparison of behavioral estimates of 
overt performance, using a task eliciting the spatial-numerical association of response codes (SNARC) effect (Cipora \& Nuerk, 2013; Dehaene et al., 1993; Wood, Willmes, Nuerk, $\&$ Fischer, 2008), and manipulating ITI. The SNARC effectshorter response times (RTs) to small numbers using the left rather than the right hand and the opposite for large numbers - is held to reflect the association between number magnitude and location of the response hand. The SNARC effect is a well-replicated behavioral effect (Basso Moro, Dell'Acqua, \& Cutini, 2017; Fischer \& Shaki, 2014; Wood et al., 2008). Our choice of a SNARC task was not unmotivated, since no fMRI study so far has clearly established the brain regions selectively modulated by the SNARC effect (Weis, Estner, Krick, Reith, \& Lachmann, 2015), with only one fNIRS study pointing to the intraparietal sulcus and left angular gyrus as possible neural substrates of the SNARC effect (Cutini et al., 2012).

In keeping with the general idea that ITI length should not determine major discrepancies between the results of behavioral (Short-ITI) and neuroimaging (Long-ITI) studies employing analogous designs, our predictions can be summarized as follows. Mapelli, Rusconi, and Umiltà (2003) have shown a progressive increment of the SNARC effect magnitude as RTs lengthened, a finding that is explicitly predicted by the computational model put forth by Gevers, Verguts, Reynvoet, Caessens, and Fias (2006). Given that longer RTs are usually observed in Long-ITI (e.g., Sohn, Ursu, Anderson, Stenger, \& Carter, 2000) relative to Short-ITI designs (e.g., Rogers \& Monsell, 1995), we expected an increment of the SNARC effect magnitude in the Long-ITI condition of the present study. Other than this, we expected ITI to not affect a set of variables that we selected to extend our exploration to a range of accessory psychological aspects, such as perceived task difficulty and/or anxiety.

\section{Materials and methods}

\section{Participants}

Thirty-six students at the University of Padova (right-handed, 24 females; mean age 19.5 years, range: 19-24 years, all with normal or corrected-to-normal vision) participated in the experiment after providing written informed consent. No participant reported a history of neurological/psychiatric disorder or was under medication at the time of testing. The study was approved by the ethical committee of the University of Padova (Protocol Number 2226).

\section{Stimuli and procedures}

The experiment took place in two sessions, at approximately 3 weeks apart. During each session, participants were seated in a comfortable chair placed inside a sound-attenuated and dimly lit room, at a distance of $57 \mathrm{~cm}$ from a 17-in. LCD monitor with a black background. Participants were instructed to rest their index fingers on the ' $\mathrm{D}$ ' and ' $\mathrm{K}$ ' keys of the computer keyboard throughout the entire session, which was subdivided in two blocks. At the end of the first block, participants were invited to take a short break. As shown in Fig. 1, each trial began with the presentation of a fixation cross $\left(0.45^{\circ}\right)$ for 300 $\mathrm{ms}$, followed by a white digit $\left(1.48^{\circ} \times 0.97^{\circ}\right)$ ranging from 1 to 9 (except 5). In the first block, participants had to press the ' $D$ ' key if the number was odd and the ' $K$ ' key if it was even. In the second block, participants had to perform the same parity judgment task, but the stimulus-response mapping was inverted. The order of stimulus-response mapping was counterbalanced across participants. The digit was displayed for a maximum of $2 \mathrm{~s}$, and replaced with a blank screen upon response detection. Following a response, two possible ITIs could have occurred, counterbalanced across sessions and across stimulus-response mapping order. In one session, 1,100-1,500 ms (randomly jittered in steps of $100 \mathrm{~ms}$, Short-ITI) elapsed before the presentation of the central fixation cross for the next trial (Dehaene et al., 1993; Georges, Hoffmann, \& Schiltz, 2016; Hoffmann, Pigat, \& Schiltz, 2014; but see also Cipora \& Nuerk, 2013; Nuerk, Wood, \& Willmes, 2005), whereas in the other session 6,000$10,000 \mathrm{~ms}$ elapsed (randomly jittered in steps of 1,000 ms, Long-ITI; see Cutini et al., 2012). After $5 \mathrm{~min}$ of practice, participants performed a sequence of 640 trials (in the case of Short-ITI) or 256 trials (in the case of Long-ITI). The design included compatible SNARC trials (when participants were required to press the key with the left index finger and the number was smaller than 5 or when they were required to press the key with the right index finger and the number was larger than 5) and incompatible SNARC trials (when the opposite occurred). The design was balanced with respect to SNARC compatibility and/or response code repetition trials (trials with the same SNARC compatibility and/or in which the response code was used as in the preceding trial) and SNARC compatibility and/or response code alternation trials (trials with an inverted SNARC compatibility and/or in which the response code had to be changed with respect to the preceding trial; Liefooghe, Verbruggen, Vandierendonck, Fias, \& Gevers, 2007). The paradigm was created using E-Prime software (Psychology Software Tools Inc ${ }^{\circledR}$, Pittsburgh, PA, USA).

At the beginning and at the end of each session, participants completed the 20-items questionnaire State-Trait Anxiety Inventory [STAI] Form Y (Spielberger, Gorsuch, Lushene, Vagg, \& Jacobs, 1983) in order to evaluate the state of anxiety (i.e., conscious, immediate, and transitory emotional state, such as feelings of unease, worry, tension, and/or stress; Catell \& Scheier, 1961) before and after the parity judgment task. At the end of each session, participants completed the DP-15 rating scale (Delignières, 1993) to investigate the 


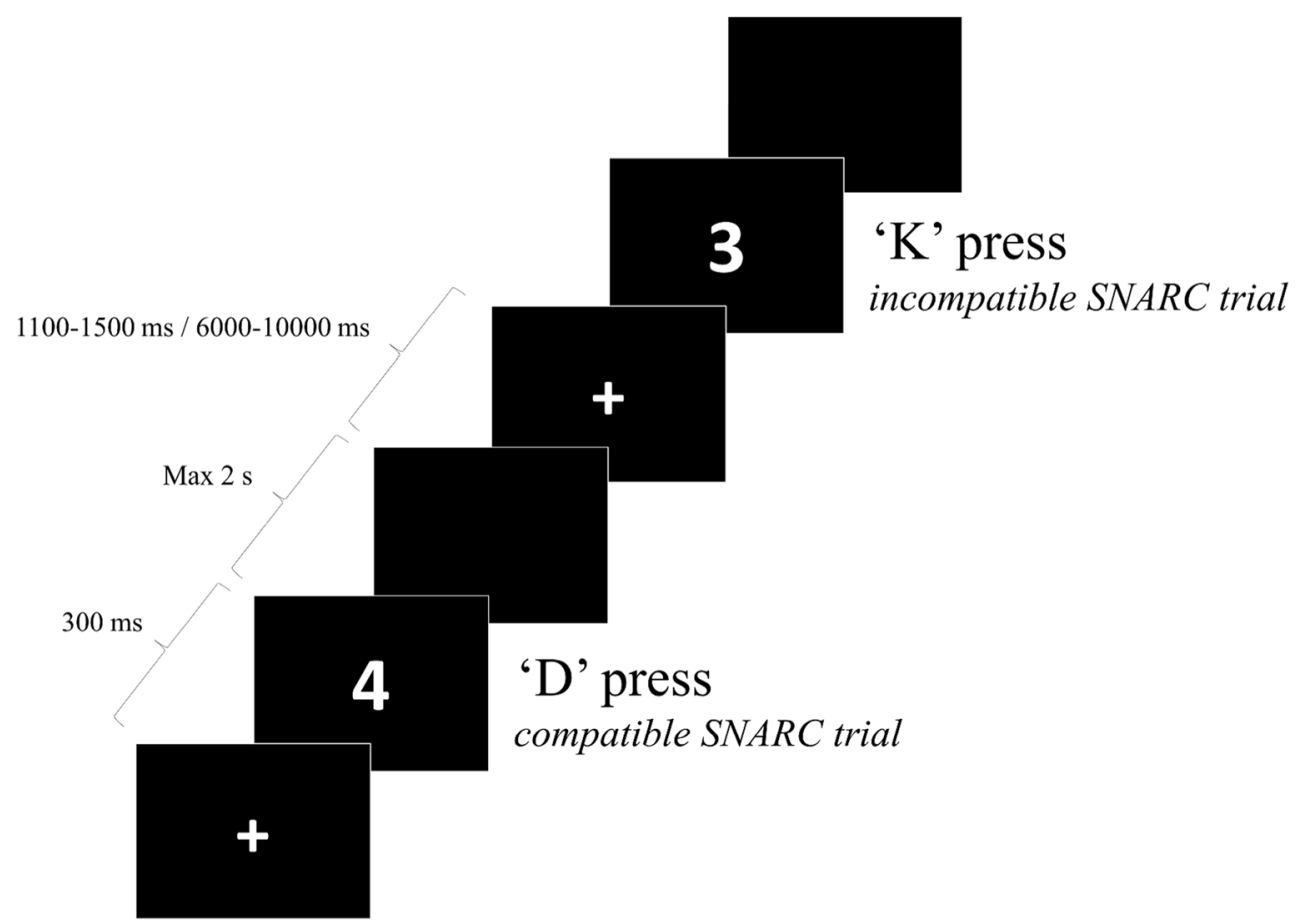

Fig. 1 Schematic illustration of the experimental protocol. In this example, the participant was required to press the ' $D$ ' key if the number was even and the ' $\mathrm{K}$ ' key if the number was odd. In one session, the ITI

perceived task difficulty, attributing to the task a value from 1 to 15 (where 1 corresponded to very, very easy; 6 to easy; 10 to difficult; 15 to very, very difficult). All participants completed the Edinburgh Handedness Inventory (Oldfield, 1971), assessing hand dominance, to exclude left-handed participants.

\section{Results}

\section{Standard analysis}

Participants' responses were scored in terms of reaction times (RTs) and accuracy. Two out of 36 participants were excluded from analyses since they were right-handed for less than 0.5 (proportion), according to the Edinburgh Handedness Inventory (Oldfield, 1971).

Accuracy values were submitted to a $2 \times 2$ mixed ANOVA, considering ITI (short vs. long) and SNARC (compatible vs. incompatible) as within-subjects factors, and session order (Short-ITI Session 1, Long-ITI Session 2 vs. Long-ITI Session 1, Short-ITI Session 2) as a between-subjects factor. Participants were more accurate to respond to SNARC compatible trials than SNARC incompatible trials (.96 vs. .95), $F(1,32)=4.41, \eta_{\mathrm{p}}{ }^{2}=.121, p=.044$. No effect of session order, $F(1,32)=.17, \eta_{\mathrm{p}}^{2}=.005, p=.687$, or ITI, $F(1,32)=$ $.31, \eta_{\mathrm{p}}^{2}=.010, p=.580$, was found. was short (i.e., $1,100-1,500 \mathrm{~ms}$ ), while in the other session the ITI was long $(6,000-10,000 \mathrm{~ms})$

Only RTs associated with a correct response and with values between 200 and 1,500 ms were analyzed and submitted to a $2 \times 2$ mixed ANOVA, considering ITI (short vs. long) and SNARC (compatible vs. incompatible) as within-subjects factors, and session order as a between-subjects factor. The Greenhouse-Geisser correction was applied when appropriate, and the false discovery rate correction was applied in all $t$ tests that followed the ANOVAs to correct for multiple comparisons. Participants were faster when ITI was short (537.15 vs. 596.18$), F(1,32)=20.86, \eta_{\mathrm{p}}{ }^{2}=.395, p<.001$, and when trials were SNARC compatible (560.73 vs. 572.58$), F(1,32)$ $=13.86, \eta_{\mathrm{p}}{ }^{2}=.302, p=.001$. Furthermore, the interaction between ITI and SNARC, $F(1,32)=4.49, \eta_{\mathrm{p}}{ }^{2}=.123, p=$ .042 , highlighted the presence of a statistically significant SNARC effect - estimated by subtracting RTs in compatible trials from RTs in incompatible trials - only in the Long-ITI session, $t(33)=-3.61, p=.001$ (see Fig. 2). No effect of session order was found, $F(1,32)=.38, \eta_{\mathrm{p}}^{2}=.012, p=.540$.

To better qualify this factors' interaction, we analyzed RTs in each of the four cells of the orthogonal combination of ITI (short vs. long) and session number (first vs. second) separately with four paired-sample $t$ tests. In the first session, independently of ITI, a statistically significant SNARC effect was found at the group level, $t(16)=$ $-2.27, p=.037$, for Short-ITI; $t(16)=-3.23, p=.005$, for Long-ITI. In the second session, instead, independently of ITI, no SNARC effect was found, $t(16)=-0.32, p=.756$, for Short-ITI; $t(16)=-1.83, p=.086$, for Long-ITI. It is 


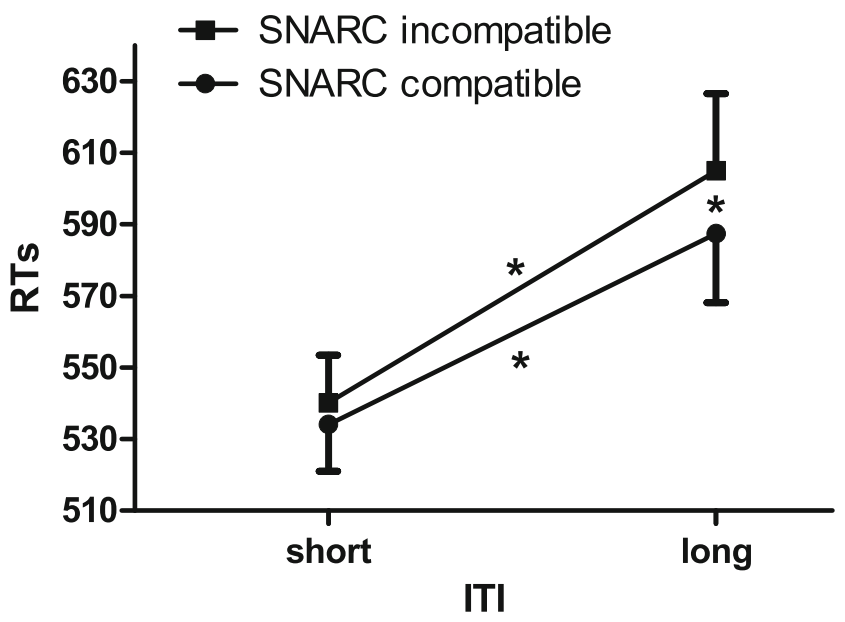

Fig. 2 Interaction between ITI and SNARC. The Long-ITI induced longer RTs for both compatible and incompatible SNARC trials, and the SNARC effect was significant only when the ITI was long. Error bars indicate standard errors

worth noting that while the SNARC effect in the second session for the Short-ITI design is far from being significant, it tends to significance for the Long-ITI design. Of note, four Bayesian paired-sample $t$ tests performed with JASP software (JASP Team, 2017), using a normal distribution $(M=0, S D=.707)$ as prior, corroborated these results indicating the following Bayes Factors: $B F_{10}=$ 2.62 for Short-ITI Session $1, B F_{10}=13.11$ for Long-ITI Session $1, B F_{10}=0.34$ for Short-ITI Session 2, and $B F_{10}$ $=1.36$ for Long-ITI Session 2 .

\section{Analysis with equal number of trials}

Since the number of trials in the Short-ITI session was almost 3 times the number of trials in the Long-ITI one, further analyses were carried out on the Short-ITI data only, to exclude the possibility that the smaller SNARC effect was due to higher habituation. Two scenarios were investigated, separately for Short-ITI Sessions 1 and 2: (1) the first 64 trials for each SNARC compatibility condition (a total of 256 trials) were extracted and their RTs averaged (early trials analysis); (2) 64 trials for each SNARC compatibility condition were randomly extracted from the 640 trials and their RTs averaged (random trials analysis). RTs were submitted to paired-sample $t$ tests for both Short-ITI Sessions 1 and 2 and for each of the two scenarios. For both scenarios and sessions, no SNARC effect was found, thus ruling out the habituation hypothesis, $t(16)=-1.09, p=.291$, for Session 1 , early trials analysis; $t(16)=-0.65, p=.523$, for Session 2, early trials analysis; $t(16)=-1.72, p=.104$, for Session 1 , random trials analysis; $t(16)=.17, p=.867$, for Session 2 , random trials analysis.

\section{Task difficulty, state anxiety, and test-retest reliability}

The mean rating of the perceived task difficulty was $6.2 \pm 1.8$ in the Short-ITI session, and $5.9 \pm 1.9$ in the Long-ITI session, corresponding both to easy (Delignières, 1993). The anxiety rate before and after the task was submitted to a $2 \times 2$ mixed ANOVA, considering ITI (short vs. long) and time of measurement (before vs. after the experiment) as within-subjects factors, and session order as a between-subjects factor. A statistically significant interaction between ITI and time of measurement, $F(1,33)=6.7, \eta_{\mathrm{p}}{ }^{2}=.173, p=.014$, revealed a different state anxiety after the experiment for Short-ITI and Long-ITI sessions (see Fig. 3). While participants did not modify their state anxiety after a Long-ITI session, they demonstrated a significant increase in state anxiety after a ShortITI session, $t(33)=-2.84, p=.032$. No significant correlation was however found between the SNARC effect and the difference in state anxiety between time of measurements.

The reliability of the participants' performance in the two sessions was investigated through Spearman correlations between RTs for compatible/incompatible trials in the two sessions for both group orders. Significant correlations were found in Short-ITI Session 1 versus Long-ITI Session 2 between RTs associated with compatible $\left(r_{\mathrm{s}}=.82, p<.001\right)$ and incompatible $\left(r_{\mathrm{s}}=.83, p<.001\right)$ trials, as well as in Long-ITI Session 1 versus Short-ITI Session 2 between RTs associated with compatible $\left(r_{\mathrm{s}}=.86, p<.001\right)$ and incompatible $\left(r_{\mathrm{s}}=.86\right.$, $p<.001)$ trials.

\section{Discussion}

As in other very well-established phenomena within the experimental cognitive psychology, such as, for example, the foreperiod effect, where the duration and variability of

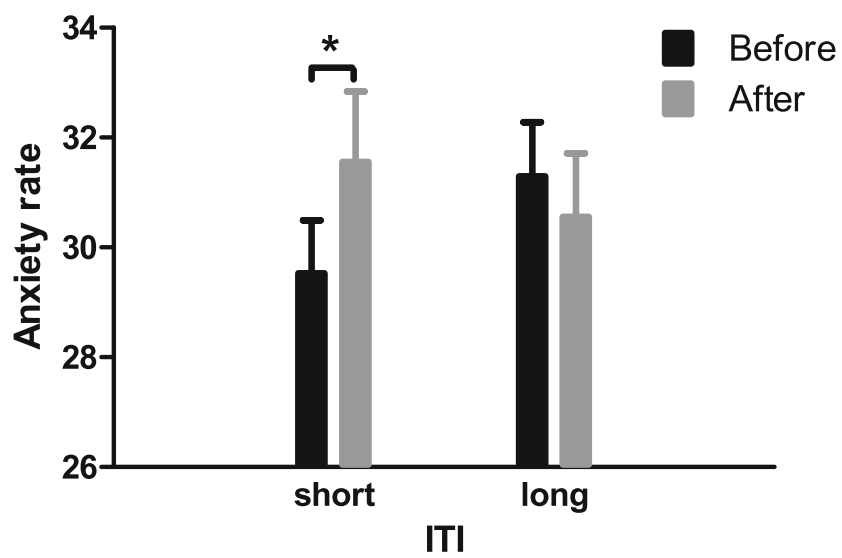

Fig. 3 Interaction between ITI and state anxiety before and after the experiment. Short-ITI induced a significant increase in the state anxiety. Error bars represent standard errors 
the interval between a warning and the actual stimulus influences RTs (Niemi \& Näätänen, 1981), ITI duration influences RTs. Our results, furthermore, highlighted that not only ITI modulated RTs, it directly influenced the SNARC effect. Although the SNARC effect was replicated for the Short-ITI design when performed in the first session as expected, in the second session the SNARC effect disappeared. Furthermore, it showed a higher tendency toward significance in the Long-ITI session with respect to the Short-ITI one. This result, along with the absence of SNARC effect when equalizing the number of trials of the Short-ITI design to the Long-ITI design, points to a stronger influence of longer ITIs on modulating the SNARC effect. Although the stronger SNARC effect in Long-ITI sessions might be explained by their longer RTs, our results seem to point to a primary role of ITI in the modulation of this effect. The presence of a SNARC effect in the first session, both for Short-ITI and Long-ITI designs, and the lack of significance of the SNARC effect at equalized number of trials, reveals that in Short-ITI experiments, a higher number of trials is required to isolate the SNARC effect; this result might indicate either the presence of higher noise in the participant's responses to the task or a higher attentive state of the participant, given the speeded sequence of stimuli, so that more trials are required for the effect to emerge. However, the lack of SNARC effect in the random trials analysis seems to point to the former as most likely explanation. Furthermore, the correlations between RTs associated with the compatible/incompatible trials in the two sessions suggest that participants had a comparable level of performance during the two experimental sessions, independently of ITI and/or session order.

ITI duration seems also to impact the replicability of the SNARC effect: When the Short-ITI design was performed after the Long-ITI one, the SNARC effect totally disappeared, whereas when the opposite occurred, the SNARC effect was reduced but with a tendency to significance. These results cannot be explained with habituation alone: In that case, Long-ITI in the second session should have been the most affected (i.e., 640 trials in the first session and 256 trials in the second one vs. the opposite scenario).

Interestingly, ITI duration also affects the state anxiety of the participants. In Long-ITI sessions, participants reported no changes in their state anxiety before and after task execution, whereas an increase in state anxiety was reported by participants after a Short-ITI session. This difference in the participant's emotional state while executing a Short-ITI versus Long-ITI experiment has two important implications: first, the brain activity and sympathetic response will likely differ in the two experiments (Kirilina et al., 2012; Minati, Kress, Visani, Medford, \& Critchley, 2011); second, the attentive state of the participant will likely differ, given that state anxiety has been found to be associated with an overfunctioning of the alerting network (Pacheco-Unguetti, Acosta, Callejas, \& Lupiáñez, 2010). Furthermore, this different state anxiety might explain the longer RTs in Long-ITI compared to ShortITI designs.

Our results, therefore, demonstrate that ITI has a strong impact on the SNARC effect, as well as the state anxiety of the participants, thus raising important concerns on the comparability between neuroimaging and behavioral studies, including the divergence of results between behavioral and fMRI studies of the SNARC effect.

In the case of the SNARC effect, the present findings seem to indicate that at a behavioral level, it might be worthier to perform "more relaxed" experiments, with longer ITIs and fewer trials, instead of stressing participants with a lot of trials at high presentation rates. It is worth noting that other behavioral effects might not be influenced by ITI, or ITI might impact their time course differently than with the SNARC effect. We might suppose, for instance, that Long-ITI designs might be detrimental for the Simon effect, since previous studies showed a reversed Simon effect with longer RTs (Mapelli et al., 2003; Schroeder, Pfister, Kunde, Nuerk, \& Plewnia, 2016). Further studies could investigate whether an ideal ITI for both neuroimaging and behavioral experiments can be found, to enhance study comparability and to further reduce participant's stress and time required by the experiment. It should also be noted that while in behavioral experiments jittering the ITI is not required, neuroimaging experiments usually vary ITI duration randomly, to avoid time locking physiological confounds to stimuli onset. Future studies should investigate whether having a fixed versus jittered ITI could also influence psychological effects.

Our results, although only behavioral, allow us also to reason about the implication of ITI duration on the experimental paradigms often employed in fMRI/fNIRS. There are four main types of designs employed in fMRI studies: block, event-related, fast event-related, and mixed designs (Amaro $\&$ Barker, 2006). The main difference among them is ITI duration. Block designs consist of a serial presentation of stimuli of the same category (i.e., a block), with ITI similar to behavioral studies. Differences between experimental conditions are investigated by comparing different blocks. Although this design should induce in the participant the same behavior as in behavioral studies, the measured brain response will be given by the contribution of all stimuli in a block, also with an influence of incorrect responses. Furthermore, whereas in behavioral studies stimulus categories are randomly presented during the experiment, in block designs stimuli of the same category should be presented within a block, thus raising questions on the comparability of the neuropsychological results of these two designs. Event-related designs allow the temporal characterization of the hemodynamic response in each trial. These designs are the most similar to behavioral studies, the only difference being a longer ITI duration, as 
presented in the current study. Our results indicate that these two designs are actually not fully comparable, given the different state anxiety and participant's behavior when the same paradigm, but with different ITI, is used. In fast event-related designs, ITI is shorter than the duration of the elicited hemodynamic response, thus reducing the length of the experiment but at the expenses of dealing with the nonlinearity of the neurovascular coupling in overlapping hemodynamic responses (Friston, Josephs, Rees, \& Turner, 1998). A common rule of thumb is to vary ITI during the study to allow deconvolution, keeping a minimum of $4 \mathrm{~s}$ between consecutive stimuli (Glover, 1999), an ITI longer than that of behavioral studies. Further studies should evaluate the comparability between fast event-related and behavioral studies to also understand how a randomly varying ITI influences behavioral results. Finally, in mixed designs, each block of stimuli of the same category contains a fast event-related design. Although this interesting design allows for the characterization of both transient and sustained neural activity (Petersen \& Dubis, 2012), the highly variable ITI duration and the different way of presenting stimuli compared to behavioral studies raise some concerns on the link between the neural activation and the actual behavioral effect. Further studies are required to evaluate the combined effect of ITI duration and stimulus presentation style on behavioral effects.

Regardless of the design employed, our results should warn researchers interested in testing cognitive theories with neuroimaging data (Coltheart, 2013) to carefully consider ITI duration as a factor in the modulation of cognitive performance. Our results suggest that it might be worthier to perform a behavioral experiment before the neuroimaging one, with the exact same design, for a prior evaluation of the participant's behavioral performance.

Acknowledgements This work was supported by Grant STPD 11B8HM from the University of Padova.

\section{References}

Amaro, E., \& Barker, G. J. (2006). Study design in fMRI: Basic principles. Brain and Cognition, 60, 220-232.

Basso Moro, S., Dell'Acqua, R., \& Cutini, S. (2017). The SNARC effect is not a unitary phenomenon. Psychonomic Bulletin \& Review.

Brigadoi, S., Cutini, S., Meconi, F., Castellaro, M., Sessa, P., Marangon, M., ... Dell'Acqua, R. (2017). On the role of the inferior intraparietal sulcus in visual working memory for lateralized single-feature objects. Journal of Cognitive Neuroscience, 29, 337-351.

Catell, R. B., \& Scheier, I. H. (1961). The meaning and measurement of neuroticism and anxiety. Oxford, England: Ronald.

Cipora, K., \& Nuerk, H.-C. (2013). Is the SNARC effect related to the level of mathematics? No systematic relationship observed despite more power, more repetitions, and more direct assessment of arithmetic skill. The Quarterly Journal of Experimental Psychology, 66, 1974-1991.
Coltheart, M. (2013). How can functional neuroimaging inform cognitive theories? Perspectives on Psychological Science, 8, 98-103.

Cutini, S., Scarpa, F., Scatturin, P., Dell'Acqua, R., \& Zorzi, M. (2012). Number-space interactions in the human parietal cortex: Enlightening the SNARC effect with functional near-infrared spectroscopy. Cerebral Cortex, 24, 444-451.

Cutini, S., Scatturin, P., Basso Moro, S., \& Zorzi, M. (2014). Are the neural correlates of subitizing and estimation dissociable? An fNIRS investigation. Neurolmage, 85(Pt. 1), 391-399.

Dehaene, S., Bossini, S., \& Giraux, P. (1993). The mental representation of parity and number magnitude. Journal of Experimental Psychology: General, 122, 371-396.

Delignières, D. (1993). La perception de l'effort et de la difficulté [Perception of effort and difficulty]. In J.P. Famose (Ed.), Cognition et Performance (pp. e183-218). Paris: INSEP

Fischer, M. H., \& Shaki, S. (2014). Spatial associations in numerical cognition-From single digits to arithmetic. Quarterly Journal of Experimental Psychology, 67, 1461-1483.

Friston, K. J., Josephs, O., Rees, G., \& Turner, R. (1998). Nonlinear event-related responses in fMRI. Magnetic Resonance in Medicine, 39, 41-52.

Georges, C., Hoffmann, D., \& Schiltz, C. (2016). How math anxiety relates to number-space associations. Frontiers in Psychology, 7, 1401.

Gevers, W., Verguts, T., Reynvoet, B., Caessens, B., \& Fias, W. (2006). Numbers and space: A computational model of the SNARC effect. Journal of Experimental Psychology: Human Perception and Performance, 32, 32-44.

Glover, G. H. (1999). Deconvolution of impulse response in event-related BOLD fMRI. NeuroImage, 9, 416-429.

Hoffmann, D., Pigat, D., \& Schiltz, C. (2014). The impact of inhibition capacities and age on number-space associations. Cognitive Processing, 15, 329-342.

JASP Team. (2017). JASP (Version 0.8.3.1). https://jasp-stats.org/. Accessed 1 Nov 2017

Kirilina, E., Jelzow, A., Heine, A., Niessing, M., Wabnitz, H., Brühl, R., ... Tachtsidis, I. (2012). The physiological origin of task-evoked systemic artefacts in functional near infrared spectroscopy. NeuroImage, 61, 70-81.

Liefooghe, B., Verbruggen, F., Vandierendonck, A., Fias, W., \& Gevers, W. (2007). Task switching and across-trial distance priming are independent. European Journal of Cognitive Psychology, 19, 1-16.

Luck, S. J., \& Vogel, E. (1997). The capacity of visual working memory for features and conjunctions. Nature, 390, 279-281.

Mapelli, D., Rusconi, E., \& Umiltà, C. (2003). The SNARC effect: An instance of the Simon effect? Cognition, 88, B1-B10.

Minati, L., Kress, I. U., Visani, E., Medford, N., \& Critchley, H. D. (2011). Intra- and extra-cranial effects of transient blood pressure changes on brain near-infrared spectroscopy (NIRS) measurements. Journal of Neuroscience Methods, 197, 283-288.

Niemi, P., \& Näätänen, R. (1981). Foreperiod and simple reaction time. Psychological Bulletin, 89, 133-162.

Nuerk, H.-C., Wood, G., \& Willmes, K. (2005). The universal SNARC effect: The association between number magnitude and space is amodal. Experimental Psychology, 52, 187-194.

Oldfield, R. C. (1971). The assessment and analysis of handedness: The Edinburgh inventory. Neuropsychologia, 9, 97-113.

Pacheco-Unguetti, A. P., Acosta, A., Callejas, A., \& Lupiáñez, J. (2010). Attention and anxiety: Different attentional functioning under state and trait anxiety. Psychological Science, 21, 298-304.

Petersen, S. E., \& Dubis, J. W. (2012). The mixed block/event-related design. NeuroImage, 62, 1177-1184.

Revkin, S. K., Piazza, M., Izard, V., Cohen, L., \& Dehaene, S. (2008). Does subitizing reflect numerical estimation? Psychological Science, 19, 607-614. 
Rogers, R. D., \& Monsell, S. (1995). Costs of a predictible switch between simple cognitive tasks. Journal of Experimental Psychology: General, 124, 207-231.

Schroeder, P. A., Pfister, R., Kunde, W., Nuerk, H.-C., \& Plewnia, C. (2016). Counteracting implicit conflicts by electrical inhibition of the prefrontal cortex. Journal of Cognitive Neuroscience, 28, $1737-1748$.

Sohn, M. H., Ursu, S., Anderson, J. R., Stenger, V. A., \& Carter, C. S. (2000). The role of prefrontal cortex and posterior parietal cortex in task switching. Proceedings of the National Academy of Sciences, 97, 13448-13453.
Spielberger, C. D., Gorsuch, R. L., Lushene, P. R., Vagg, P. R., \& Jacobs, A. G. (1983). Manual for the State-Trait Anxiety Inventory. Palo Alto: Consulting Psychologists Press.

Weis, T., Estner, B., Krick, C. M., Reith, W., \& Lachmann, T. (2015). SNARC meets SPARC in fMRI-Interdependence of compatibility effects depends on semantic content. Neuropsychologia, 77, 331-338.

Wood, G., Willmes, K., Nuerk, H.-C., \& Fischer, M. H. (2008). On the cognitive link between space and number: A meta-analysis of the SNARC effect. Psychology Science Quarterly, 4, 489-525. 